\title{
Multiple return times in the quantum baker map
}

\author{
M. Fannes ${ }^{\mathrm{a} *}$, P. Spincemaille ${ }^{\mathrm{a} \dagger}$

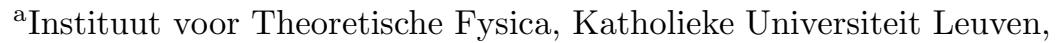 \\ Celestijnenlaan 200D, B-3001 Heverlee, Belgium
}

For chaotic classical systems, the distribution of return times to a small region of phase space is universal. We propose a simple tool to investigate multiple returns in quantum systems. Numerical evidence for the baker map and kicked top points, also in the quantum case, at a universal distribution.

PACS : 05.45.Mt,05.45.Pq

keywords : return times, baker map, kicked top

\section{Introduction}

In classical dynamical systems, one may study how long it takes a system to return to the phase space region it occupied at time zero. In general, this time will increase when the phase space area under consideration is decreased. M. Kac has shown that, for an ergodic dynamical system, the average return time, assuming that the system starts in a set $A$, is given by $\mu(A)^{-1}$ where the average is taken over the ergodic measure $\mu$. Related to this are results about asymptotically rare events. Consider a sequence $A_{k}$ of subsets of phase space such that $\lim _{k \rightarrow \infty} \mu\left(A_{k}\right)=0$. If the time for a point $x$ in phase space to visit $A_{k}$ is denoted by $\tau_{k}(x)$, then the question arises how the product $\mu\left(A_{k}\right) \tau_{k}(x)$ behaves. This turns out, for a large class of models, to have an exponential distribution [5].

One may wonder whether a similar situation occurs in quantum mechanics. In finite dimensional systems, looking with increasing precision (corresponding to the $k \rightarrow \infty$ limit of above) means increasing the dimension of the system, which amounts to taking its classical limit. Here, the inverse dimension plays the rôle of Planck's constant. Return times were studied for various quantum dynamical systems. A natural way of measuring returns is provided by the notion of

\footnotetext{
*Email: mark.fannes@fys.kuleuven.ac.be

${ }^{\dagger}$ Email: pascal.spincemaille@fys.kuleuven.ac.be

¥Acknowledges financial support from FWO project G.0239.96
}

transition probability: for any two vectors $\varphi$ and $\psi$ in a Hilbert space it is defined as $|\langle\varphi \mid \psi\rangle|^{2}$.

\section{Multiple return times}

If a system starts from an initial state $\varphi_{0}$, one can consider

$$
n \in \mathbb{N} \mapsto\left\langle\varphi_{0} \mid \varphi_{n}\right\rangle \in \mathbb{C},
$$

where $\varphi_{n}$ is the state of the system after $n$ time steps. For finite dimensional systems this function is almost periodic. Interesting behaviour is displayed only when looking at the correct time scale. It is known that, for classically chaotic systems, the quantum system follows its classical counterpart in the sense of coherent states up to a certain breaking time of the order of $\log N$, where $N$ is the dimension of its Hilbert space of states. We propose to analyse the function (11) through the spectrum of the Gram matrix associated with a sequence of normalised vectors $\varphi_{0}, \cdots, \varphi_{K-1}$ :

$$
G_{K}=\left(\begin{array}{ccc}
\left\langle\varphi_{0} \mid \varphi_{0}\right\rangle & \cdots & \left\langle\varphi_{0} \mid \varphi_{K-1}\right\rangle \\
\vdots & \ddots & \vdots \\
\left\langle\varphi_{K-1} \mid \varphi_{0}\right\rangle & \cdots & \left\langle\varphi_{K-1} \mid \varphi_{K-1}\right\rangle
\end{array}\right) .
$$

$G_{K}$ is a positive semi-definite matrix and its eigenvalues are independent of the order of the vectors and of any extra phase added to any of them.

Information about the amount and the frequency of vectors that return to a small neighbourhood 
in Hilbert space lies encoded in the spectrum of this matrix. This fact is best appreciated by examining a simple example. Consider the sequence $\left\{\psi_{1}, \psi_{2}, \psi_{2}, \psi_{1}, \psi_{3}, \psi_{4}, \psi_{1}\right\}$, where the $\psi_{i}$ 's are normalised and mutually orthogonal. This amounts to specifying a sequence of letters belonging to a given alphabet (the classical case). The Gram matrix consists of only zeroes and ones (because of the orthonormality) and its spectrum is $\{0,0,0,1,1,2,3\}$. An eigenvalue different from zero indicates the number of times a certain vector is repeated and its multiplicity tells how many vectors are repeated that often. In our example, only two vectors are repeated, the vector $\psi_{1}$ twice and $\psi_{2}$ once, summing up to three, which is exactly the multiplicity of zero. This example provides the link with the return times mentioned in the introduction: one considers a partition of phase space and keeps track of the different sets a particle visits during its evolution. The associated Gram matrix has a one for its $i j$ th entry if the visited sets at times $i$ and $j$ are the same and a zero otherwise. Hence calculating the spectrum of the Gram matrix immediately gives all the return times.

In general, for a true quantum system, the vectors in a sequence will not be orthogonal and the overlaps will be complex numbers with absolute value smaller than or equal to one. However, a similar interpretation of the spectrum can be obtained remembering that overlaps with absolute value close to 1 indicate vectors close to each other, while overlaps close to zero indicate almost orthogonal vectors. Generically, the non-zero eigenvalues of the Gram matrix are non-degenerate and the notion of multiplicity of above should be replaced by the spectral density, i.e. a degenerate eigenvalue is now replaced by different eigenvalues close to each other. The multiplicity of zero is equal to the number of linear dependencies in the given sequence. Two rather extreme cases will clarify this. A large concentration of eigenvalues around 1 indicates that a large amount of vectors have small mutual overlaps. This can be compared with the classical situation of above, with a spectrum consisting mainly of ones and only a few zeroes, indicating very few repetitions. A presence of many eigenvalues close to zero sug- gests that many vectors lie close to one another, leading to many large overlaps. This compares to a classical situation where some letters are repeated many times, giving rise to many zeroes in the spectrum and only a few large integers.

A point that has not been stressed yet is the number of time steps to consider. The spectrum of the Gram matrix will surely contain an eigenvalue zero when the number $K$ of vectors exceeds the dimension $N$ of the Hilbert space. Including too many time steps causes a large degeneracy of zero. We shall demonstrate, using numerical simulations, that also $K$ has to be taken large such that the ratio $K / N$ is a fixed number $\tau$ : time measured on the Heisenberg scale. The same scaling is relevant for a random model that can be treated analytically.

A natural choice for the initial state is a coherent state centred around a point in classical phase space. There are several reasons for this. Taking the large $N$ limit immediately implies the comparison of quantum systems with different dimensions. This also entails that initial states for different dimensions have to be comparable. Coherent states exist for every $N$, occupy a volume $1 / N$ of phase space and have a clear limit, namely a single point in phase space. Also, a coherent state is typically not a eigenvector of the evolution which would lead to a completely trivial spectrum of the Gram matrix.

The precise object we shall investigate is the empirical eigenvalue distribution of the Gram matrix. If a matrix has eigenvalues $\lambda_{1}, \cdots, \lambda_{K}$, then its empirical eigenvalue distribution is

$$
\rho(\mathrm{d} \lambda) \equiv \sum_{\mathrm{i}=1}^{\mathrm{K}} \delta\left(\lambda-\lambda_{\mathrm{i}}\right) \mathrm{d} \lambda .
$$

In this way, the number of eigenvalues in a set $\Lambda \subset \mathbb{R}$ is given by $\rho(\Lambda)$.

We shall illustrate this approach by example. We consider two simple quantum dynamical systems frequently encountered in the quantum chaos literature. They have clearly defined classical limits. The first example is the quantum baker map and the second the kicked top. 


\section{Models}

The quantum baker map is a dynamics on $\mathbb{C}^{N}$ with $N$ even [1]. Defining the discrete Fourier transform on $\mathbb{C}^{M}$

$$
F_{M}|m\rangle=\frac{1}{M} \sum_{n=0}^{M-1} \mathrm{e}^{\frac{2 \pi i n m}{M}}|n\rangle,
$$

the unitary evolution operator of the baker map is

$$
U=F_{N}\left(\begin{array}{cc}
F_{N / 2}^{-1} & 0 \\
0 & F_{N / 2}^{-1}
\end{array}\right)
$$

Its classical limit can be shown to be

$$
\left(\begin{array}{c}
q \\
p
\end{array}\right) \mapsto\left(\begin{array}{c}
2 q \bmod 1 \\
p / 2+[2 q] / 2
\end{array}\right)
$$

where $\left(\begin{array}{l}q \\ p\end{array}\right)$ is a point in the phase space $[0,1] \times$ $[0,1]$. The position and momentum $q$ and $p$ are the classical limits of

$$
Q_{N}|m\rangle=\frac{m}{N}|m\rangle \quad \text { and } \quad P_{N}=F_{N} Q_{N} F_{N}^{-1} .
$$

The baker map is an example of a chaotic system: it has a positive Lyapunov exponent equal to $\log 2$.

First, we numerically construct a coherent state in an $N$-dimensional Hilbert space centred around a chosen classical point in phase space. One way of doing this is by calculating the ground state of an appropriate Harper operator and subsequently shifting it over to the desired classical point. Then, we apply the operator $U$ a number of times to calculate the overlaps needed in the Gram matrix. Note that when the first row of the Gram matrix is calculated, all other matrix elements are known because of the unitarity of the evolution. Unitarity has another interesting consequence: the precision of the calculation does not exponentially decrease with the number of time steps. One way to see this, is applying the unitary evolution to an initial state many times, followed by applying an equal number of times the adjoint of the evolution. The resulting state is remarkably close to the original state, despite the presence of numerical round-off errors in the components of the vectors and the unitary matrix. This is in sharp contrast with numerical simulations of chaotic classical systems where positive Lyapunov exponents cause the numerical errors to quickly exceed the desired precision. The eigenvalues of the Gram matrix are calculated and the empirical eigenvalue distribution is represented by the bar diagrams of Figure 1. The solid black line will be commented on later in this letter.

The first thing that Figure 11 reveals is the time scale $N$ on which a non-trivial eigenvalue density is present. For a fixed time on the Heisenberg time scale, i.e. a fixed ratio $\tau=K / N$, the eigenvalue densities are very similar. For comparison, choosing $K$ of the order of $\log N$ produces a Gram matrix close to the identity matrix and therefore a spectrum which is greatly concentrated around one. For times much larger than $N$ the linear dependencies dominate the picture and the empirical eigenvalue distribution tends to a Dirac distribution at zero. Secondly, we note that we did not specify which coherent state we began with. Our simulations show that the initial state does not affect the form of the eigenvalue distribution. Diagrams, very similar to those of Figure 1 1 , were obtained for different initial states.

The same procedure was carried out for a second example: the quantum kicked top. This is a quantum spin- $j$ system with basic observables $J_{x}, J_{y}$ and $J_{z}$ obeying the usual commutation relations $\left[J_{x}, J_{y}\right]=i J_{z}+$ cycl. perm. . The unitary evolution is given by

$$
U=\mathrm{e}^{-\mathrm{i} k J_{z}^{2} / 2 j} \mathrm{e}^{-\mathrm{i} p J_{y}} .
$$

The classical limit of this system is a dynamics on the two dimensional sphere given by

$$
\begin{aligned}
x^{\prime}= & (x \cos p+z \sin p) \cos (k z \cos p-k x \sin p) \\
& +y \sin (k z \cos p-k x \sin p), \\
y^{\prime}= & (x \cos p+z \sin p) \sin (k z \cos p-k x \sin p) \\
& -y \cos (k z \cos p-k x \sin p), \\
z^{\prime}= & -x \sin p+z \cos p,
\end{aligned}
$$

with $(x, y, z)$ a unit vector in $\mathbb{R}^{3}$ which is the classical limit of $\left(J_{x}, J_{y}, J_{z}\right) / j$. This map is a com- 


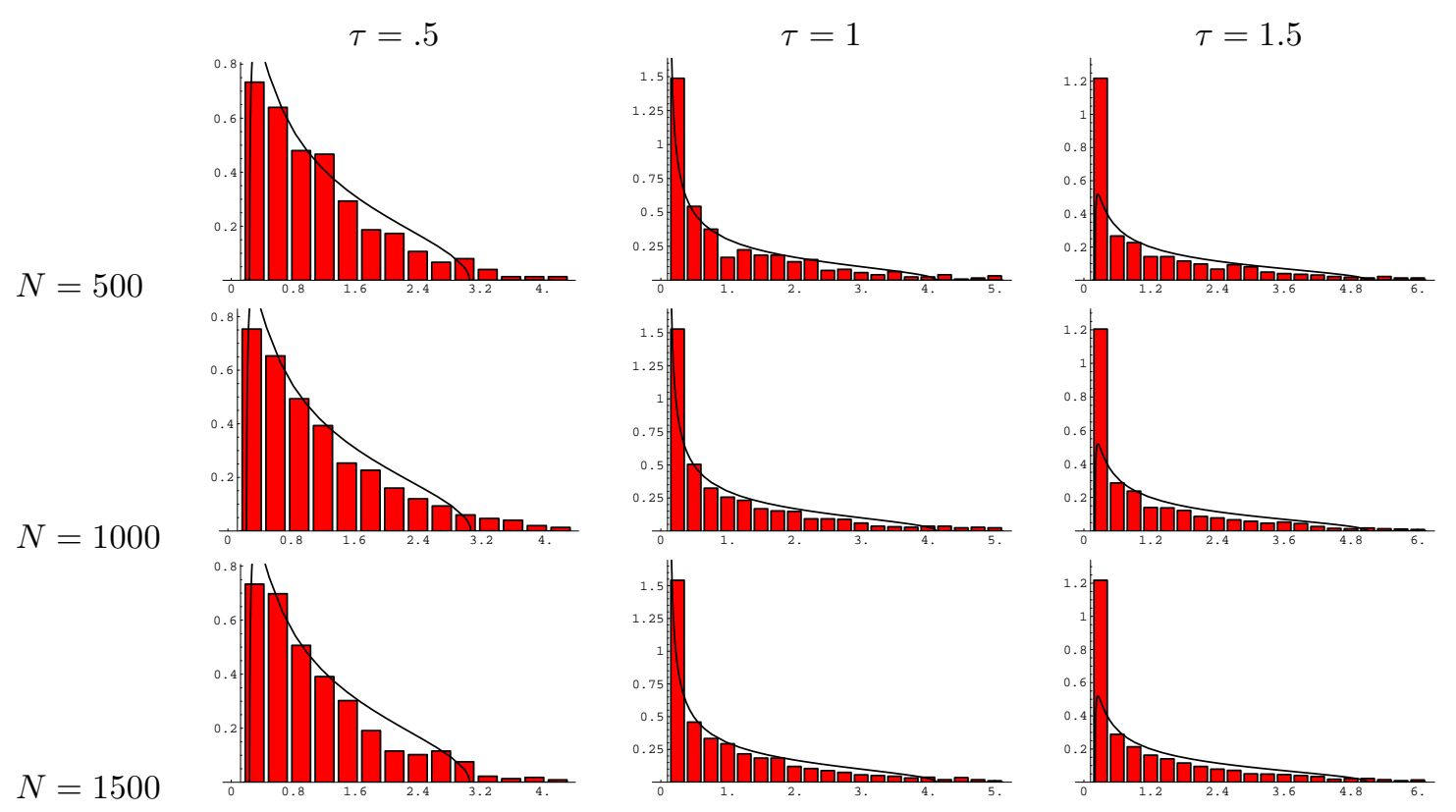

Figure 1. Eigenvalue density of Gram matrices for the quantum baker map for various values of $N$ and $\tau=K / N$

position of a rotation around the y-axis over an angle $p$ and a torsion around the $z$-axis over an angle proportional to the $z$-component. Numerical studies suggest the following behaviour. For small values of the parameters $k$ and $p$, this system displays regular behaviour in large parts of the sphere, separated by chaotic regions. The regular islands shrink as the parameters grow, leading eventually to almost global chaos (See [《]). This is an interesting feature of this model: varying the control parameters $k$ and $p$ changes the behaviour of the classical model considerably. It can be concluded from Figure 2 that this difference in behaviour is also present in the quantum kicked top. For small values of the control parameters $k$ and $p$ there are a lot of approximate returns during the first $K=\tau N$ time steps. This is clear from the large number of eigenvalues close to zero. We expect that in the limit $K=\tau N \rightarrow \infty$ this distribution tends to a degenerate one in zero as the system visits only a small fraction of the available states. Enlarging the control parame- ters places more weight on higher eigenvalues and a totally different eigenvalue distribution appears. These distributions appear to be very similar to the ones for the baker map for the same value of $\tau$.

One unexplained feature in the diagrams is the solid line. This is actually the graph of an analytical function obtained in the following way. Instead of trying to calculate the limiting empirical eigenvalue distribution for a true quantum mechanical model, like the baker map or the kicked top, one can try to simplify the calculations by considering a random vector model. For an $N$ dimensional Hilbert space, a sequence of $K$ normalised vectors is chosen randomly and independently according to the uniform distribution on the unit sphere. In this way, one can try to mimic the evolution of a system which wanders wildly in Hilbert space. The limiting eigenvalue distribution $(N, K \rightarrow \infty$ and $K / N \rightarrow \tau)$ of the Gram matrix associated with this sequence of random vectors can be calculated explicitly and is given 


$$
k=1.5 \text { and } p=1
$$
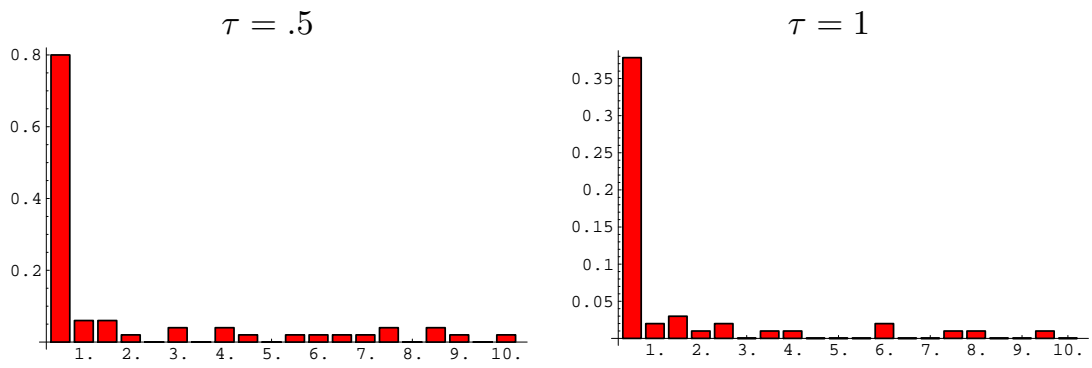

$k=6.5$ and $p=1.5$
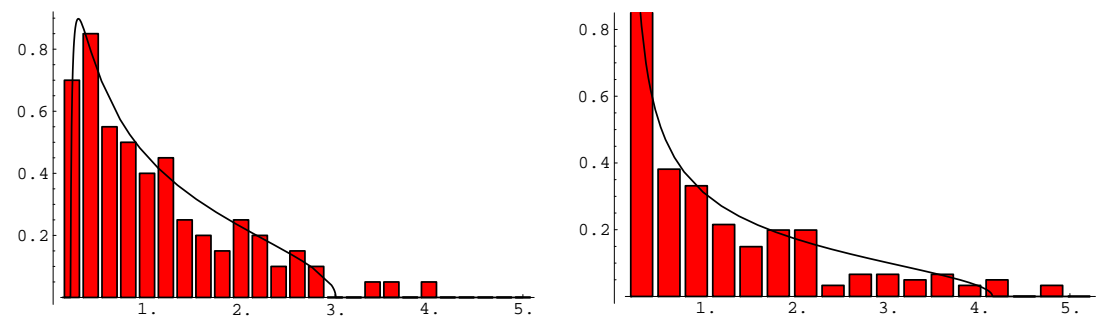

Figure 2. Eigenvalue density of Gram matrices for the quantum kicked top for various values of $\tau, k$ en $p(\tau$ is defined as the ratio $K / N$ with $N=2 j+1=201)$

by the Marchenko-Pastur distribution [3]

$$
\rho_{\tau}(\mathrm{d} t)=\left\{\begin{array}{lr}
\frac{\sqrt{4 \tau t-(t+\tau-1)^{2}}}{2 \pi \tau t} \mathrm{~d} t & 0<\tau \leq 1 \\
\frac{\tau-1}{\tau} \delta(t) \mathrm{d} t & \\
+\frac{\sqrt{4 \tau t-(t+\tau-1)^{2}}}{2 \pi \tau t} \mathrm{~d} t & 1<\tau .
\end{array}\right.
$$

This density is the solid line depicted in Figures 1 and 2. A possible explanation for the similarity between the eigenvalue distributions generated by the baker map and the kicked top and the Marchenko-Pastur distribution is that, when the number of time steps is linear in the dimension of the Hilbert space, subsequent vectors in a chaotic quantum evolution are almost random and visit all of the Hilbert space without preference for one direction or region.

\section{Conclusion}

The eigenvalue distribution of the Gram matrix appears to be a meaningful object in the so-called deep quantum regime where the system is allowed to visit a finite fraction of the total number of available states. Moreover, for systems with a chaotic classical limit, numerical simulations indicate that the limiting distribution is universal and coincides with that of random vectors, even if the latter model is not a unitary dynamics. If the classical limit is regular, we find a degenerate distribution. Linking the limiting spectrum of the Gram matrix with the distribution of energy or quasi-energy level separations [2] definitely deserves further research. Another interesting question, that is also relevant in quantum information theory, is what happens when the dynamical system is no longer isolated but weakly coupled to an environment. In such a situation, density matrices instead of vector states must be used to describe the decoherence induced by the environment.

\section{REFERENCES}

1. N.L. Balazs and A. Voros, The Quantized Baker's Transformation, Ann. Phys. 190, 131 (1989)

2. M.V. Berry and M. Tabor, Level clustering in the regular spectrum, Proc. Roy. Soc. A 356, 
375-394 (1977)

3. M. De Cock, M. Fannes, and P. Spincemaille, On quantum dynamics and statistics of vectors, J. Phys. A: Math. Gen. 32, 6547-6571 (1999)

4. F. Haake, M Kuś, and R. Scharf, Classical and Quantum Chaos for a Kicked Top, Zeitschrift für Physik B65, 381-395 (1987)

5. M. Hirata, B. Sausol and S. Vaienti, Statistics of return times: a general framework and new applications, Commun. Math. Phys. 206, 33-55 (1999) 